1 in the second week to detect chronic stage abnormalities. (Kidokoro $\mathrm{H}$ et al. Pediatrics Oct 2009;124:e468-e475). (E-mail: kidokoro@kosei.anjo.aichi.jp).

Dr Joseph J Volpe, in an editorial, comments that the report by Kidokoro and associates shows that the EEG may be important in diagnosis, timing, and severity of PVL (Pediatrics Oct 2009;124:e542-e544), but the infants in this study were a severely affected subset of the premature population. In general, MRI in the neonatal period is the most effcctive method of identifying white-matter injury in premature infants. Ultrasonography is useful in detection of severe injury. The timing of the insult by EEG is useful in the decision to order potentially protective interventions such as antioxidants etc.

\title{
EFFECT OF NEONATAL SEIZURES ON COGNITIVE OUTCOME OF HYPOXIC-ISCHEMIC ENCEPHALOPATHY
}

The independent effect of clinical neonatal seizures and their treatment on longterm neurodevelopmental outcome in 77 term newborns at risk for hypoxic-ischemic encephalopathy (HIE) was determined in a study at University of California San Francisco. Clinical seizures were recorded and graded prospectively assigning points $(0-10)$ for frequency, status, medications, and EEG abnormal background and epileptiform discharges. Eleven children (14.3\%) had severe neonatal seizures (composite seizure score $>4$ ), 14 (18.2\%) had mild/moderate seizures (score 1 to 3 ), and $52(67.5 \%)$ had no seizures (seizure score 0 ). Of the 25 infants with seizures, EEG was abnormal in 14 (56\%). The severity of HIE measured by MRI was most highly associated with cognitive outcome, measured by WPPSI-R and neuromotor score, at age 4 years. The pattern of the HIE correlated with severity of seizures $(\mathrm{P}<0.0001)$; basal nuclei predominant patern was associated with severe seizures, and the watershed pattern with mild/moderate seizures. Children with severe seizures had a lower FSIQ than those with mild/moderate seizures $(\mathrm{P}<0.0001)$. (Glass HC, Glidden D, Jeremy RJ, Barkovich AJ, Ferriero DM, Miller SP. Clinical neonatal seizures are independently associated with outcome in infants at risk for hypoxic-ischemic bnrain injury. J Pediatr September 2009;155:318-323). (Respond: Hannah C Glass MDCM, University of California San Francisco, Department of Neurology, Box 0663, 521 Parnassus Ave, C-215, San Francisco, CA 94143. E-mail: Hannah.Glass@ucsf.edu).

COMMENT. The authors conclude that clinical neonatal seizures with birth asphyxia are associated with worse neurodevelopmental outcome, independent of the severity of hypoxic-ischemic brain injury. The effect of the seizures themselves could not be differentiated from the cognitive effects of treatment with phenobarbital and phenytoin, however. Also, almost half of patients with clinical seizures had a normal EEG. Seizure severity was graded by frequency, medication use, and EEG, but not by seizure pattern. According to Volpe JJ (N Engl J Med 1973;289:413; also, In Gluck L. Editor. Intrauterine Asphyxia and the Developing Fetal Brain. Chicago. Year Book Med Pub, 1977), virtually all infants with HIE related seizures have "subtle" seizures. Subtle seizures are manifested by 1) tonic horizontal deviation or jerking of eyes; 2) eye-lid blinking or fluttering; 3) sucking, smacking of lips; 4) 'swimming" or "rowing" movements of limbs; and/or 5) apneic spells. Infants also exhibit multifocal clonic seizures or decerebrate/decorticate tonic seizures in 
addition to subtle seizures, but not generalized tonic-clonic seizures, the type expected to result in brain injury.

Subtle seizures described as "breast-stroke swimming movements" were previously reported in studies of seizure patterns in newborn animals (Millichap JG. Proc Soc Exp Biol and Med 1957;96:125-129). Transient opisthotonus, tremors, and clonic movements were also characteristic of newborn seizure patterns, but in rats aged 1 to 15 days subjected to graded electroshock, a generalized tonic clonic seizure could not be elicited. Failure to induce convulsions in the newborn rat was associated with a low level of carbonic anhydrase in the brain. The maximal seizure pattern was correlated with increasing age and the higher maturational levels of carbonic anhydrase in the brain of older animals. Observation of the newborn seizure pattern in addition to seizure frequency and EEG discharges might permit closer correlation with severity of HIE and outcome. If neonatal seizures do contribute to HIE brain injury, inhibition of the development of brain carbonic anhydrase would be expected to lessen the severity of neonatal seizures and result in improved neurodevelopmental outcome. Detailed EEG monitoring is essential for confirmation of diagnosis of neonatal seizures, especially subtle seizures.

\section{DEVELOPMENTAL CORRELATES OF MICROCEPHALY}

Developmental and motor function at age 2 years of 958 children born before the $28^{\text {th }}$ week of gestation were assessed at Boston and Harvard Universities and other centers, comparing those with microcephaly at birth or 2 years with children with normal head circumference. A total of $11 \%$ of infants in the sample had microcephaly at 2 years. Microcephaly at 2 years, but not at birth, was predictive of severe motor and cognitive impairments at 2 years. Of children with congenital microcephaly, $71 \%$ had normal head circumference at 2 years and similar neurodevelopmental outcomes to those with normal head circumference at birth and 2 years. Among children with microcephaly at 2 years, more than half had a Mental Developmental Index $<70$, and almost a third had cerebral palsy, rates 3 times greater than among children without microcephaly. Neonatal cranial ultrasound showing white matter damage increased risk of poor neurodevelopmental outcome. (Kuban KCK, Allred EN, O'Shea TM, et al. Developmental correlates of head circumference at birth and two years in a cohort of extremely low gestational age newborns. J Pediatr Sept 2009;155:344-349). (Response: Karl Kuban MD, SMEpi, One Boston Medical Center Place, Dowling 3 South, Boston, MA 02118. E-mail: karl.kuban@bmc.org).

COMMENT. Extremely low gestational age newborns (in the ELGANs epidemiological study) are at risk of neurodevelopmental dysfunction and autism (Kuban K. J Pediatr 2009;154:535-540). Microcephaly at 2 years, but not at birth, is associated with cognitive and motor impairment at age 2. Almost three-fourths of ELGANs with congenital microcephaly outgrow the problem by age 2 years. Congenital microcephaly is only a risk factor for $\mathrm{CP}$ or cognitive impairment if the microcephaly persists.

\section{CAUSES OF NEONATAL HYPOGLYCEMIC BRAIN INJURY}

Perinatal factors associated with hypoglycemic brain injury were studied by review of medical records in 60 hypoglycemic neonates at Tottori University, Yonago, Japan. Patients 